\title{
General properties of the evolution of unstable states at long times
}

\author{
K. Urbanowski* \\ University of Zielona Gora, Institute of Physics, \\ ul. Prof. Z. Szafrana 4a, 65-516 Zielona Gora, Poland.
}

October 28, 2018

\begin{abstract}
An effect generated by the nonexponential behavior of the survival amplitude of an unstable state at the long time region is considered. It is known that this amplitude tends to zero as $t$ goes to the infinity more slowly than any exponential function of $t$. Using methods of asymptotic analysis we find the asymptotic form of this amplitude in the long time region in a general model independent case. We find that the long time behavior of this amplitude affects the form of the instantaneous energy of unstable states: This energy should be much smaller for suitably long times $t$ than the energy of this state for $t$ of the order of the lifetime of the considered unstable state.
\end{abstract}

PACS: 03.65.-w, 03.65.Ta, 11.10.St,

Keywords: unstable states, nonexponential decay, long time deviations.

\section{Introduction}

Unstable states $|u\rangle$ of the system under considerations are characterized by their decay law, $\mathcal{P}_{u}(t)$,

$$
\mathcal{P}_{u}(t)=|a(t)|^{2},
$$

*email: K.Urbanowski@proton.if.uz.zgora.pl 
where

$$
a(t)=\langle u \mid u(t)\rangle,
$$

is the probability amplitude of finding the system at the time $t$ in the initial state $|u\rangle \in \mathcal{H}$ prepared at time $t_{0}=0, \mathcal{H}$ is the Hilbert space of states of the system, $\||u\rangle \|=1$ so $a(0)=1$, and $|u(t)\rangle \in \mathcal{H}$ solves the Schödinger equation

$$
i \hbar \frac{\partial}{\partial t}|u(t)\rangle=H|u(t)\rangle, \quad|u(0)\rangle=|u\rangle,
$$

where $H$ denotes the total selfadjoint Hamiltonian for the system. From basic principles of quantum theory it is known that the nondecay amplitude $a(t)$, and thus the decay law $\mathcal{P}_{u}(t)$ of the unstable state $|u\rangle$ decaying in the vacuum, are completely determined by the density of the energy distribution $\omega(\varepsilon)$ for the system in this state [1],

$$
a(t)=\int_{-\infty}^{+\infty} \omega(\varepsilon) e^{-\frac{i}{\hbar} \varepsilon t} d \varepsilon
$$

where $\omega(\varepsilon) \geq 0$.

Note that (4) and (2) together with the condition that $|u\rangle$ is a normalized vector mean that there must be

$$
a(0)=\int_{-\infty}^{+\infty} \omega(\varepsilon) d \varepsilon=1
$$

From the last property one concludes that $\omega(\varepsilon)$ is an absolutely integrable function, $\omega(\varepsilon) \in L_{1}(-\infty, \infty)$. So the amplitude $a(t)$ is the Fourier transform of $\omega(\varepsilon)$ (see (4)) and thus from the Riemann-Lebesgue Lemma it follows that $a(t)$ must tend to zero as $t \rightarrow \infty$ [1, 2].

A condition, which is necessary on physical grounds, that $H$ has a spectrum bounded from below, Spec. $(H)=\left[E_{\text {min }},+\infty\right)$, and $E_{\min }>-\infty$, reduces the set of functions $\omega(\varepsilon) \in L_{1}(-\infty, \infty)$ to such $\omega(\varepsilon) \in L_{1}(-\infty, \infty)$ that $\omega(\varepsilon)=0$ for $\varepsilon<E_{\text {min }}$ and $\omega(\varepsilon) \geq 0$ for $\varepsilon \geq E_{\text {min }}$. Thus in fact the integration in (4), (5) and in similar formulae is taken over $\varepsilon \in\left[E_{\text {min }},+\infty\right)$.

In [3] assuming that the spectrum of $H$ must be bounded from below and using the Paley-Wiener Theorem [4] it was proved that in the case of unstable states there must be

$$
|a(t)| \geq A e^{-b t^{q}},
$$


for $|t| \rightarrow \infty$. Here $A>0, b>0$ and $0<q<1$. This means that the decay law $\mathcal{P}_{u}(t)$ of unstable states decaying in the vacuum, (1), can not be described by an exponential function of time $t$ if time $t$ is suitably long, $t \rightarrow \infty$, and that for these lengths of time $\mathcal{P}_{u}(t)$ tends to zero as $t \rightarrow \infty$ more slowly than any exponential function of $t$. The analysis of the models of the decay processes shows that $\mathcal{P}_{u}(t) \simeq e^{-\frac{\gamma_{u}^{0} t}{\hbar}}$, (where $\gamma_{u}^{0}$ is the decay rate of the state $|u\rangle)$, to a very high accuracy for a wide time range $t$ : From $t$ suitably greater than some $T_{0} \simeq t_{0}=0$ but $T_{0}>t_{0}\left(\mathcal{P}_{u}(t)\right.$ has nonexponential power-like form for short times $t \in\left(t_{0}, T_{0}\right)$ - see, e.g. [3, [5, 6] ]) up to $t \gg \tau_{u}=\frac{\hbar}{\gamma_{u}^{0}}$ and smaller than $t=t_{a s}$, where $t_{a s}$ denotes the time $t$ for which the long time nonexponential deviations of $a(t)$ begin to dominate (see eg., [3], [5] [10]). From this analysis it follows that in the general case the decay law $\mathcal{P}_{u}(t)$ takes the inverse power-like form $t^{-\lambda}$, (where $\lambda>0$ ), for suitably large $t \geq t_{a s} \gg \tau_{u}$ [3], 5] - 7]. This effect is in agreement with the general result (6). Effects of this type are sometimes called the "Khalfin effect" (see eg. [11]).

The problem how to detect possible deviations from the exponential form of $\mathcal{P}_{u}(t)$ in the long time region has been attracting attention of physicists since the first theoretical predictions of such an effect [12, 13, 8]. The tests that have been performed over many years to examine the form of the decay laws for $t \gg \tau_{u}$ have not indicated any deviations from the exponential form of $\mathcal{P}_{u}(t)$ in the long time region. Nevertheless, conditions leading to the nonexponetial behavior of the amplitude $a(t)$ at long times were studied theoretically [14] - 22]. Conclusions following from these studies were applied successfully in experiment described in [23, where the experimental evidence of deviations from the exponential decay law at long times was reported. This result gives rise to another problem which now becomes important: if and how the long time deviations from the exponential decay law depend on the model considered (that is, on the form of $\omega(\varepsilon)$ ), and if (and how) these deviations affect the energy of the unstable state and its decay rate in the long time region. 


\section{General long time properties of the nonde- cay amplitude}

Many fundamental and general model independent properties of the nondecay amplitude $a(t)$ follow from the fact that $a(t)$ is the Fourier transform of an absolutely integrable function $\omega(\varepsilon)$. So, if one assumes that all derivatives $\omega^{(k)}(\varepsilon),(k=0,1, \ldots, n)$, exist and that $\omega^{(k)}(\varepsilon) \in L_{1}(-\infty, \infty)$ for all these $k$, (where $\omega^{(0)}(\varepsilon)=\omega(\varepsilon)$ ), then analyzing general properties of the Fourier transforms it is easy to find that for $t \rightarrow \infty$

$$
|a(t)| \leq \frac{C}{t^{n}}
$$

where $0<C<\infty$ (see [2]). So if the derivative of $\omega(\varepsilon)$ exists and $\omega^{(1)}(\varepsilon) \in$ $L_{1}(-\infty, \infty)$ then taking into account relation (6) the following estimation follows

$$
\frac{C}{t} \geq|a(t)| \geq A e^{-b t^{q}}
$$

as $t \rightarrow \infty$.

Much more information about asymptotic properties of $a(t)$ being the Fourier transform of $\omega(\varepsilon)$ one can find if the assumption required by physics that the spectrum of $H$ is bounded from below is used, eg. by $\varepsilon=E_{\text {min }}>$ $-\infty$. This means that from this time we will consider only such $\omega(\varepsilon) \in$ $L_{1}(-\infty, \infty)$ that $\omega(\varepsilon)=0$ for $\varepsilon<E_{\text {min }}$ and $\omega(\varepsilon) \geq 0$ for $\varepsilon>E_{\text {min }}$. In this case there is

$$
a(t)=\int_{E_{\min }}^{+\infty} \omega(\varepsilon) e^{-\frac{i}{\hbar} \varepsilon t} d \varepsilon .
$$

instead of the formula (41) for the amplitude $a(t)$. Using methods of the asymptotic analysis it is not difficult to find an asymptotic expansion for large values of $t$ of the Fourier integral of this type [24, 25, 26].

Let us consider for a start relatively simple case when $\lim _{\varepsilon \rightarrow E_{\min }+} \omega(\varepsilon) \stackrel{\text { def }}{=}$ $\omega_{0}>0$. Let derivatives $\omega^{(k)}(\varepsilon),(k=0,1,2, \ldots, n)$, be continuous in $\left[E_{\text {min }}, \infty\right)$, (that is let for $\varepsilon>E_{\text {min }}$ all $\omega^{(k)}(\varepsilon)$ be continuous and all the limits $\lim _{\varepsilon \rightarrow E_{\text {min }}+} \omega^{(k)}(\varepsilon)$ exist) and let all these $\omega^{(k)}(\varepsilon)$ be absolutely integrable functions then, as can be easily verified, (see [24, 25, 26]),

$$
a(t) \underset{t \rightarrow \infty}{\sim}-\frac{i \hbar}{t} e^{-\frac{i}{\hbar} E_{\min } t} \sum_{k=0}^{n-1}(-1)^{k}\left(\frac{i \hbar}{t}\right)^{k} \omega_{0}^{(k)}
$$


where $\omega_{0}^{(k)} \stackrel{\text { def }}{=} \lim _{\varepsilon \rightarrow E_{\text {min }}+} \omega^{(k)}(\varepsilon)$.

Let us now consider a more complicated form of the density $\omega(\varepsilon)$. Namely let $\omega(\varepsilon)$ be of the form

$$
\omega(\varepsilon)=\left(\varepsilon-E_{\min }\right)^{\lambda} \eta(\varepsilon) \in L_{1}(-\infty, \infty),
$$

where $0<\lambda<1$ and it is assumed that $\eta^{(k)}(\varepsilon),(k=0,1,2, \ldots, n)$, exist

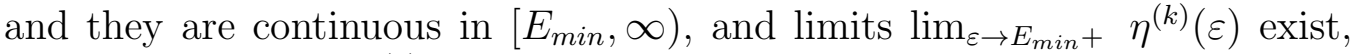
$\lim _{\varepsilon \rightarrow \infty}\left(\varepsilon-E_{\text {min }}\right)^{\lambda} \eta^{(k)}(\varepsilon)=0$ for all above mentioned $k$, then

$$
\begin{aligned}
a(t) \underset{t \rightarrow \infty}{\sim} & -\frac{i \hbar}{t} \lambda e^{-\frac{i}{\hbar} E_{\min } t}\left[\alpha_{n}(t)+\left(-\frac{i \hbar}{t}\right) \alpha_{n-1}(t)\right. \\
& +\left(-\frac{i \hbar}{t}\right)^{2} \alpha_{n-2}(t) \\
& \left.+\left(-\frac{i \hbar}{t}\right)^{3} \alpha_{n-3}(t)+\ldots\right],
\end{aligned}
$$

where (compare [25, 26])

$$
\alpha_{n-k}(t)=\sum_{l=0}^{n-k-1} \frac{\Gamma(l+\lambda)}{l !} e^{-i \frac{\pi(l+\lambda+2)}{2}} \eta_{0}^{(l+k)}\left(\frac{\hbar}{t}\right)^{l+\lambda},
$$

and $\eta_{0}^{(j)}=\lim _{\varepsilon \rightarrow E_{\text {min }}+} \eta^{(j)}(\varepsilon), \eta^{(0)}(\varepsilon)=\eta(\varepsilon)$ and $j=0,1, \ldots, n$.

The main difference between asymptotic expansions (10) and (12) is that the amplitude $a(t)$ in (10) is obtained as the Fourier transform (9) of such $\omega(\varepsilon)$ that $\omega(\varepsilon)=0$ for $\varepsilon<E_{\min }$ and $\omega\left(E_{\min }\right)>0$ whereas the expansion (12) is the asymptotic expansion of the Fourier transform (91) for another type $\omega(\varepsilon)$ : namely for $\omega(\varepsilon)$ such that $\omega\left(E_{\min }\right)=0$ (see (11)).

From (10), (12) it follows that the estimation (8) is true for all physically admissible $\omega(\varepsilon)$. This estimation holds for $t \rightarrow \infty$, strictly speaking for $t>t_{a s}$. For $t \sim \tau_{u}, t<t_{a s}$ the nondecay amplitude $a(t)$ takes the form

$$
a(t) \simeq e^{-i \frac{t}{\hbar}\left(E_{u}^{0}-\frac{i}{2} \gamma_{u}^{0}\right)},
$$

to a very high accuracy [3], [5] - [11, [14] - 22]. In this formula $E_{u}^{0}$ denotes the measured energy of the unstable particle described by the state-vector $|u\rangle$. There is $E_{u}^{0}>E_{\text {min }}$. 
For the energy densities $\omega(\varepsilon)$ leading to the asymptotic form of the amplitude $a(t)$ of type (10) the time $t_{a s}$ can be found by comparing the square of the modulus of the amplitude $a(t)$ from the relation (14) and the square of the modulus of the leading component of (10). So $t_{a s}$ can be found by solving the following transcendental equation

$$
e^{-\frac{\gamma_{u}^{0}}{\hbar} t}=\hbar^{2}\left(\frac{\omega\left(E_{\min }\right)}{t}\right)^{2} .
$$

This means that the value of $t_{a s}$ depends on the model considered: it depends on the density $\omega(\varepsilon)$ and on the $\gamma_{u}^{0}$. If $\omega(\varepsilon)$ has the form (11) then a similar method can be used to find a corresponding equation for $t_{a s}$.

\section{Energy of unstable states at long time re- gion}

Using the Khalfin's estimation (6) of the decay law (1) one can examine the asymptotic properties of the decay rate $\gamma_{u}$ of an unstable state $|u\rangle$. In a general case the decay rate $\gamma_{u}$ equals,

$$
\gamma_{u}=\gamma_{u}(t) \stackrel{\text { def }}{=}-\frac{\hbar}{\mathcal{P}_{u}(t)} \frac{\partial \mathcal{P}_{u}(t)}{\partial t} .
$$

From (16), (11) and (14) one infers that

$$
\gamma_{u}=\gamma_{u}(t) \equiv \gamma_{u}^{0}, \quad\left(\text { for } t<t_{a s}\right),
$$

which is obvious. From (6) it follows that in the asymptotic case $t \rightarrow \infty$ the decay rate $\gamma_{u}(t)$ can not be larger than [27],

$$
\gamma_{u}(t) \underset{t \rightarrow \infty}{\sim} b q t^{-\mu},
$$

where $\mu \equiv 1-q>0$. So for $t>t_{a s}$ one finds that $\left.\gamma_{u}(t)\right|_{t \rightarrow \infty}<\gamma_{u}^{0}$ for every physically admissible $\omega(\varepsilon)$, and in general that $\lim _{t \rightarrow \infty} \gamma_{u}(t)=0$.

The problem is how the energy $E_{u}$ of the unstable state $|u\rangle$ behaves for $t>t_{a s}$. The solution of this problem follows from the observation that the amplitude $a(t)$ can be found either by solving the Schrödinger equation (3) 
or using the equation for the projection of the state vector ( see [27] and references one can find therein), which in the case of one-dimensional subspace $\mathcal{H}_{\|}$of states $\mathcal{H}$ spanned by the normalized vector $|u\rangle$ has the following simple form

$$
i \hbar \frac{\partial a(t)}{\partial t}=h_{u} a(t), \quad a(0)=1,
$$

where $h_{u}$ is the "effective Hamiltonian" for the one-dimensional subspace of states $\mathcal{H}_{\|}$. In general, $h_{u}$ can depend on time $t, h_{u} \equiv h_{u}(t)$ [27, 28]. One meets this effective Hamiltonian when one starts with the Schrödinger Equation (3) for the total state space $\mathcal{H}$ and looks for the rigorous evolution equation for the distinguished subspace of states $\mathcal{H}_{\|} \subset \mathcal{H}$. There are many approximate methods to calculate $h_{u}$ [27] but taking into account the problem raised above their use is not necessary. It is sufficient to use the property that the exact effective Hamiltonian $h_{u}(t)$ must fulfill the following identity [27]

$$
h_{u} \equiv h_{u}(t) \stackrel{\text { def }}{=} i \hbar \frac{\partial a(t)}{\partial t} \frac{1}{a(t)} .
$$

Direct application (20) to the relation (14) yields

$$
h_{u}(t)=h_{u}^{0} \equiv E_{u}^{0}-\frac{i}{2} \gamma_{u}^{0}, \quad\left(\text { for } t<t_{a s}\right),
$$

which could be expected. Note that from (16) and (20) it follows that simply

$$
\gamma_{u}(t)=-2 \Im\left(h_{u}(t)\right)
$$

Similarly, the real part of $h_{u}(t)$ is the instantaneous energy, $E_{u}(t)$, of the system in the state $|u\rangle$ under considerations

$$
E_{u} \equiv E_{u}(t)=\Re\left(h_{u}(t)\right)
$$

(Here $\Re(z)$ and $\Im(z)$ denote the real and imaginary parts of $z$ respectively).

Note that relations (20) and (19) establish a direct connection between the amplitude $a(t)$ for the state $|u\rangle$ and the exact effective Hamiltonian $h_{u}(t)$ governing the time evolution in the one-dimensional subspace $\mathcal{H}_{\|} \ni|u\rangle$. Thus the use of the relation (20) is one of the most effective tools for the accurate analysis of the early- as well as the long-time properties of the instantaneous energy and decay rate for a given qausistationary state $|u(t)\rangle$. 
Now let us analyze the asymptotic properties of $h_{u}(t)$ for $t \rightarrow \infty$. For the densities $\omega(\varepsilon)$ leading to asymptotic expansion (10) one finds that for $t \rightarrow \infty$,

$$
\begin{aligned}
& i \hbar \frac{\partial a(t)}{\partial t} \simeq \quad E_{\min } a(t)+\left(\frac{i \hbar}{t}\right)^{2} e^{-\frac{i}{\hbar} E_{\min } t} \times \\
& \times\left\{\omega_{0}-2 \omega_{0}^{(1)}\left(\frac{i \hbar}{t}\right)+3 \omega_{0}^{(2)}\left(\frac{i \hbar}{t}\right)^{2}\right. \\
&\left.-4 \omega_{0}^{(3)}\left(\frac{i \hbar}{t}\right)^{3}+\ldots\right\} .
\end{aligned}
$$

The next step is to use the relation (20). So one should now divide (24) by (10) and then collect together all components of the same order with respect to $\left(\frac{\hbar}{t}\right)$. As the result one obtains the asymptotic form of $h_{u}(t)$ for $t \rightarrow \infty$,

$$
\begin{aligned}
\left.h_{u}^{\infty}(t) \stackrel{\text { def }}{=} h_{u}(t)\right|_{t \rightarrow \infty}= & E_{\min }-i \frac{\hbar}{t} \\
& -\frac{\omega_{0}^{(1)}}{\omega_{0}}\left(\frac{\hbar}{t}\right)^{2}+\ldots .
\end{aligned}
$$

One obtains a similar form of $h_{u}^{\infty}(t)$ for the amplitude $a(t)$ given by formulae (12), (13). Indeed starting from (12) one finds after some algebra that

$$
h_{u}^{\infty}(t)=E_{\text {min }}-c_{1} \frac{\hbar}{t}-c_{2}\left(\frac{\hbar}{t}\right)^{2}-c_{3}\left(\frac{\hbar}{t}\right)^{3}+\ldots,
$$

where $c_{1}, c_{2}, c_{3}, \ldots$ are complex numbers with negative or positive real and imaginary parts.

A surprising conclusion following from the result (25) is that in the long time region the leading component of the asymptotic form of the decay rate $\gamma_{u}(t)$ has the same form for a large class of physically admissible models,

$$
\left.\gamma_{u}^{\infty}(t) \stackrel{\text { def }}{=} \gamma_{u}(t)\right|_{t \rightarrow \infty} \equiv-2 \Im\left(h_{u}^{\infty}(t)\right) \simeq 2 \frac{\hbar}{t} .
$$

Using the relation (25) we find that in the long time region the instantaneous energy $E_{u}(t)$ takes the following form

$$
\begin{aligned}
E_{u}^{\infty}(t) & \left.\stackrel{\text { def }}{=} E_{u}(t)\right|_{t \rightarrow \infty}=\Re\left(h_{u}^{\infty}(t)\right) \\
& \simeq E_{\text {min }}-\frac{\omega_{0}^{(1)}}{\omega_{0}}\left(\frac{\hbar}{t}\right)^{2}+\ldots \neq E_{u}^{0} .
\end{aligned}
$$


As one can see for all densities $\omega(\varepsilon)$ such that $\omega_{0} \equiv \omega\left(E_{\text {min }}\right)>0$ the long time properties of the leading components of the energy $E_{u}(t)$, contrary to the properties of the decay rate $\gamma_{u}(t)$, depend on the density $\omega(\varepsilon)$. The result (28) seems to be even much more surprising than (27).

We have

$$
\lim _{t \rightarrow \infty} E_{u}^{\infty}(t)=E_{\min } .
$$

Note that the same result follows from (26) which means that the relation (29) is a model independent. Taking into account that $E_{u}^{0}>E_{\min }$ the following conclusion follows: for every model (that is for every $\omega(\varepsilon)$ ) there exists such $t_{\infty} \geq t_{a s}$ that

$$
E_{u}^{\infty}(t)<E_{u}^{0}, \quad\left(\text { for } t>t_{\infty}\right) .
$$

Note that results (25) - (30) are purely quantum effects and that they follow from basic assumptions of quantum theory.

\section{Final remarks}

The problem if the long time deviations from the exponential form of the decay law affect the energy of the decaying state has been studied in [9] using a model defined by $\omega(\varepsilon)$ having a form of the truncated Lorentz function and assuming that $E_{\text {min }}=0$. It is easy to verify that inserting into (10), (25) $E_{\text {min }}=0$ and $\omega(\varepsilon)$ used in [9] reproduces relations obtained there. Results obtained in Sec. 2 and Sec. 3 show that long time behavior of the decay law $\mathcal{P}_{u}(t)$ as well as the effective Hamiltonian $h_{u}(t)$ do not depend on a specific form of the density $\omega(\varepsilon)$ but they depend rather on general integral and analytic properties of the density $\omega(\varepsilon)$. From these results it follows that for all $\omega(\varepsilon)$ having the same integral and analytic properties the amplitudes $a(t)$ and the effective Hamiltonians $h_{u}(t)$ have the same long time behavior. So these results generalize and complete essentially analysis performed in [9].

The estimation (8) of $|a(t)|$ for $t \rightarrow \infty$ follows from basic assumption of quantum theory. Similarly, estimations (10), (12) of $a(t)$ at long time region are obtained using only very general assumptions on the form of the energy density $\omega(\varepsilon)$ and the fundamental assumption that there exists a minimal energy $E_{\min }>-\infty$ in the system under considerations (i.e. that Spec. $\left.(H)=\left[E_{\text {min }},+\infty\right)\right)$. So, they should hold for every physical system fulfilling these general requirements. In general, as it follows from the analysis 
performed in Sec. 2, two types of the long time asymptotic expansion of the amplitude $a(t)$ can be observed depending on the continuity properties of the density $\omega(\varepsilon)$ at the point $\varepsilon=E_{\text {min }}$. If the density $\omega(\varepsilon)$ is a discontinuous function of $\varepsilon$ at $\varepsilon=E_{\text {min }}: \omega\left(\varepsilon<E_{\text {min }}\right)=0$ and $\omega\left(E_{\text {min }}\right)>0$, then the long time asymptotic of $a(t)$ is given by the formula (10). A particular, typical example of such $\omega(\varepsilon)$ is the truncated Lorentzian distribution function $\omega_{L}(\varepsilon)$,

$$
\omega_{L}(\varepsilon)=\frac{N}{2 \pi} \Theta\left(\varepsilon-E_{\text {min }}\right) \frac{\gamma_{u}^{0}}{\left(\varepsilon-E_{u}^{0}\right)^{2}+\left(\frac{\gamma_{u}^{0}}{2}\right)^{2}},
$$

where $N$ is a normalization constant, $E_{u}^{0}>E_{\text {min }}$, and $\Theta(\varepsilon)=\{1$ for $\varepsilon \geq$ 0 , and 0 for $\varepsilon<0\}$. This distribution is the basis of many studies of decaying systems (see, eg. [3, 10]). Many unstable systems will have an initial state energy distribution $\omega(\varepsilon)$ that is close to $\omega_{L}(\varepsilon)$ for all $\varepsilon$ values. It has been proved [5] that in such cases the decay law $\mathcal{P}_{u}(t)$ for the system and decay law $\mathcal{P}_{u}^{L}(t)$ resulting from $\omega_{L}(\varepsilon)$ must be close to each other for all values of $t$. In general the density $\omega(\varepsilon)$ having Lorentz (Breit-Wigner) shape is known from the response of a harmonically bound elektron with a dissipative term, models of resonance behavior and many other physical problems. On the other hand, if $\omega(\varepsilon)$ has the form (11), that is if it is continuous at $\varepsilon=E_{\text {min }}$, then the long time form of the amplitude $a(t)$ is given by the relation (12). A particular case of this type density distribution is $\omega(\varepsilon)$ which can be found when one considers short-range potential models of quasi-stationary states: One can find such a density for finite--width barriers as well as delta barriers and with or without a potential inside the barier, etc. (see, eg., [15, 16, 17, 20, 21, 22] and references one can find therein). In general in the models mentioned densities $\omega(\varepsilon)$ are proportional near $E_{\text {min }}$ to the square root of the energy $\varepsilon$,

$$
\omega(\varepsilon) \sim \sqrt{\left(\varepsilon-E_{\min }\right)},
$$

for $\varepsilon \geq E_{\text {min }}$, (i.e. they correspond with $\lambda=\frac{1}{2}$ in (11) and (12)), and usually there is $E_{\min }=0$ in these models. The another example of the density of this type is the density $\omega(\varepsilon)$ obtained when one considers the decay of an unstable particle into two particles [7]. The form of long time asymptotic expansions for $a(t)$ can differ from expansions (10) and (12) for $\omega(\varepsilon)$ being discontinuous at a point (or some points $j=1,2, \ldots$, ) $\varepsilon=E_{j}>E_{\text {min }}$.

The source of the effect described by the relation (25) is the long time behavior of the amplitude $a(t)$. The relation (20) establishes a direct connection 
between the properties of the amplitude $a(t)$ and the properties of the instantaneous energy $E_{u}(t)$ and decay rate $\gamma_{u}(t)$ of the unstable $|u\rangle$ at the instant $t$, (see (21), (23),$(22))$. A possibility observe the "loss of energy" described by relations (28), (30) may arise while trying to test the long time properties of the nondecay amplitude $a(t)$ after a suitable modification of such tests. So, considering a possibility of a suitable modification of the test described in [23] in such a way that the emitted energy (frequency) of the luminescence decays could be measured which could make it possible to test relations (28), (30) seems worthwile. In general, all these long time properties of unstable states should not be expected to have an effect on laboratory processes but it seems that they can affect some long time astrophysical processes.

\section{References}

[1] S. Krylov, V. A. Fock, Zh. Eksp. Teor. Fiz. 17, 93, (1947).

[2] A. N. Kolgomorov, S. V. Fomin, Elements of the Theory of Functions and Functional Analysis, Dover Publ. Inc., 1999.

[3] L. A. Khalfin, Zh. Eksp. Teor. Fiz. 33, 1371, (1957) [Sov. Phys. — JETP 6, 1053, (1958)].

[4] R. E. A. C. Paley, N. Wiener, Fourier transforms in the complex domain, American Mathematical Society, New York, 1934.

[5] L. Fonda, G. C. Ghirardii and A. Rimini, Rep. on Prog. in Phys. 41, 587, (1978).

[6] A. Peres, Ann. Phys. 129, 33, (1980).

[7] M. L. Goldberger, K. M. Watson, Collision Theory, Willey, New York 1964.

[8] P. T. Greenland, Nature 335, 298, (1988).

[9] K. Urbanowski, Eur. Phys. J.,C 58, 151, (2008).

[10] K. M. Sluis, E. A. Gislason, Phys. Rev. A 43, 4581, (1991).

[11] D. G. Arbo, M. A. Castagnino, F. H. Gaioli and S. Iguri, Physica A 227, 469, (2000). 
[12] J. M. Wessner, D. K. Andreson and R. T. Robiscoe, Phys. Rev. Lett. 29, 1126, (1972).

[13] E. B. Norman, S. B. Gazes, S. C. Crane and D. A. Bennet, Phys. Rev. Lett. 60, 2246, (1988). E. B. Norman, B. Sur, K. T. Lesko, R.M. Larimer, Phys. Lett. B 357, 521, (1995).

[14] J. Seke, W. N. Herfort, Phys. Rev. A 38, 833, (1988).

[15] R. E. Parrot, J. Lawrence, Europhys. Lett. 57, 632, (2002).

[16] J. Lawrence, Journ. Opt. B: Quant. Semiclass. Opt. 4, S446, (2002).

[17] I. Joichi, Sh. Matsumoto, M. Yoshimura, Phys. Rev. D 58, 045004, (1998).

[18] N. G. Kelkar, M. Nowakowski and K. P. Khemchandani, Phys. Rev. C 70, 024601, (2004).

[19] M. Nowakowski, N. G. Kelkar, arXiv: 0807.5103; AIP Conf. Proc. 1030, (2008), 250.

[20] R. Santra, J. M. Shainline, Ch. H. Greene, Phys. Rev., A 71, 032703, (2005).

[21] R. G. Winter, Phys. Rev., 123, 1503, (1961).

[22] T. Jiitoh, S. Matsumoto, J. Sato, Y. Sato, K. Takeda, Phys Rev. A 71, 012109, (2005).

[23] C. Rothe, S. I. Hintschich and A. P. Monkman, Phys. Rev. Lett. 96, 163601, (2006).

[24] F. W. J. Olver, Asymtotics and special functions, Academic Press, New York 1974.

[25] A. Erdelyi, Asymtotic expansions, Dover Pub. Inc., New York 1956.

[26] E. T. Copson, Asymtotic expansions, Cambridge University Press, 1965.

[27] K. Urbanowski, Phys. Rev. A 50, 2847, (1994).

[28] L. P. Horwitz, J. P. Marchand, Helv. Phys. Acta, 42, 801, (1969). 